\title{
ALTERATION OF PACEMAKER THRESHOLD BY DRUG AND PHYSIOLOGICAL FACTORS
}

\author{
T. A. Preston and R. D. Judge \\ Cardiology Section, Veterans Administration Hospital \\ and Heart Station and Department of Internal Medicine \\ University of Michigan Medical Center \\ Ann Arbor, Mich.
}

In order to accurately measure changes in cardiac electrical threshold, as evoked by pharmacological or physiological interventions, it is necessary to define clearly the term threshold and the method of measuring it. Electrical threshold at any given time is the minimum electrical impulse necessary to stimulate the heart for the specific electrical system being used at that time. Determinants of threshold for any pacemaker system are many, and can be thought of as comprising extrinsic and intrinsic factors. Extrinsic factors, which are determined by engineering design, include electrode composition, electrode surface area, electrode impedance, and the waveform, voltage, current, and duration of the stimulus. Since the biological impedance of the heart and other body tissues is nonlinear, threshold may vary with different stimulus-voltage or current levels, and changes in stimulus duration also produce changes in threshold. Intrinsic threshold factors, more the domain of the biological scientist, include the position of the electrode on or inside the heart, the electrochemical interface between the electrode and surrounding tissue, and the intrinsic excitability of the tissue.

The electrode-tissue interface is partly extrinsic and partly intrinsic, but probably cannot be altered by external interventions after complete implantation of a pacemaker system. Similarly, with the exception of repositioning a catheter, electrode position is also fixed after permanent implantation. We are therefore usually left with only one factor that can be altered after permanent implantation of a pacemaker system, i.e., excitability of cardiac tissue.

Exit block is a term that we believe may be justifiably applied to the clinical condition in which a normal pacemaker stimulus fails to excite the heart because of an abnormally high threshold. It is apparent that any total system, employing any type of pulse generator, electrodes, and electrode position in a heart, will deliver a maximal stimulus distributed through the heart in a pattern specific for that system. It is equally apparent that if the tissue which is most easily excited by that system has an excitability threshold exceeding that of the implanted system, excitation of the heart will not result. Since the range of threshold varies greatly from patient to patient and from implant to implant for any given patient, ${ }^{1,2}$ a system in which the output is great enough that exit block is never seen is probably wasteful of energy in the majority of cases. In addition, the stimulus-to-threshold ratio may be dangerously high in such a design. Because fibrillation threshold is in the range of only 10-20 times excitation threshold, proper pacemaker design may occasionally encounter a high threshold and clinical exit block, unless the output of the power unit is so high that it always exceeds any threshold encountered.

Several situations arise in which it is clinically helpful to be able to alter the excitability of the heart relative to the output of the implanted pacemaker system. Since tissue excitability is the only factor that usually can be manipulated in totally implanted systems, alteration of threshold by drug and physiological factors is of clinical importance. Measurement of threshold changes after inter- 
TABLE 1

Effect of Electrolytes on Threshold Change

\begin{tabular}{|c|c|c|c|}
\hline Electrolyte & $\begin{array}{c}\text { No. of } \\
\text { Intravenous } \\
\text { Drops per } \\
\text { Minute }\end{array}$ & $\begin{array}{l}\text { No. of } \\
\text { Tests }\end{array}$ & $\begin{array}{l}\text { Maximal \% of } \\
\text { Threshoid Change }\end{array}$ \\
\hline Polarizing solution $\dagger$ & 50 & 4 & $+17,+32,+30,+13$ \\
\hline $\begin{array}{l}\text { Dextrose in water }(10 \% \mathrm{D} / \mathrm{W}) \\
\text { Potassium chloride }(\mathrm{KCl})\end{array}$ & 50 & 4 & $+15,0,0,+3$ \\
\hline $40 \mathrm{mEq}$ in $1,000 \mathrm{ml}$ of $10 \% \mathrm{D} / \mathrm{W}$ & 50 & 4 & $+12,+12,0,0$ \\
\hline $40 \mathrm{mEq}$ in $1,000 \mathrm{ml}$ of Ringer's & 50 & 3 & $-4,-23,-13$ \\
\hline $50 \mathrm{mEq}$ in $500 \mathrm{ml}$ of Ringer's & 50 & 3 & $-20,-44,-20$ \\
\hline \multicolumn{4}{|l|}{ Sodium chloride $(\mathrm{NaCl})$} \\
\hline $0.9 \%$ & 50 & 3 & $+7,0,+5$ \\
\hline $3 \%$ & 120 & 3 & $+56,+60,+56$ \\
\hline Calcium gluconate $(0.5 \mathrm{~g})$ & $--\ddagger$ & 4 & $+17,-15,0,0$ \\
\hline
\end{tabular}

* Changes of $16 \%$ or greater are significant.

$\dagger$ Polarizing solution is 10 units of regular insulin plus $20 \mathrm{mEq}$ of $\mathrm{KCl}$ in $500 \mathrm{ml}$ of $10 \%$ $\mathrm{D} / \mathrm{W}$.

$\ddagger$ Given over a period of five minutes.

vention can be made in various ways, but must be appropriate for the system. Measurement may be in milliamperes if the voltage, impulse duration, and waveform do not change as the stimulus intensity is changed. If an external power source is utilized, measurements can be taken from oscilloscopic pictures of the stimulus; if an implanted system is used, an external induction-coupled instrument may be employed. All measurements used in this paper will be changes in stimulus energy as a percent change from basal threshold, in totally implanted systems with myocardial electrodes. Threshold was measured with an external induction-coupled threshold analyzer. ${ }^{2}$

In the basal resting state, threshold is approximately $10-25 \%$ higher than in active states, ${ }^{3}$ so the mere act of arising in the morning can have a clinically discernible effect on pacemaker performance. We have had five patients with exit block who reported an irregular, slow heart beat after rest or sleep, and who discovered, themselves, that getting up and walking around would restore normal pacing. A corollary of this finding is that the patient should be in a basal resting state for well-controlled threshold measurements, with no change in threshold for at least 30 minutes before pharmacological intervention.

The effect of electrolyte alteration on cardiac threshold was recognized early, and treatment with potassium restored pacing in some cases. ${ }^{4}$ Our studies confirm threshold changes due to electrolyte alteration, and indirectly suggest that these changes are similar to interesting metabolic diseases affecting the skeletal musculature, namely, the periodic paralyses. Although the exact mechanism of these changes probably involves cellular membrane changes, our findings suggest that any drug intervention tends to decrease threshold if intracellular potassium is decreased in relation to extracellular potassium, and threshold is increased if the ratio of intracellular to extracellular potassium is increased (TABLE 1).

Thus, intravenous injection of a physiological solution with high concentration of $\mathrm{KCl}$ would be expected to decrease the ratio of intracellular to extracellular potassium (FIGURE 1), and in our testing it did decrease the threshold. The doses employed in all our electrolyte and drug studies did not cause serum potassium changes beyond the range of normal. Solutions tending to drive potassium into cells, e.g., insulin and glucose, resulted in significant threshold 
elevations, decreasing electrical excitability of the heart. This is not an unusual phenomenon relating only to electronic pacemaker systems, but appears to be a very basic phenomenon of muscle physiology, with an interesting correlation with well-known neuromuscular disorders. Thus, in hypokalemic familial periodic paralysis the extracellular potassium level drops sharply during the attack of paralysis, with a concomitant fall of urinary potassium and demonstration of rising intracellular potassium. During attacks, the muscles are refractory to electrical stimulation. There is, in addition, some relationship between attacks and excess carbohydrate intake, and the disorder responds to administration of potassium. ${ }^{5}$ Conversely, in hyperkalemic periodic paralysis there is apparently a very low threshold with myotonia, a condition that can be improved by administration of diuretics, which lower serum potassium.

The adrenal steroids comprise the next group of drugs known to alter threshold. Although the exact mechanism is undetermined, these agents appear to alter cellular membrane permeability, producing significant and sustained threshold changes. Whatever the mechanism may be, the effects of glucocorticoids and mineral corticoids are antagonistic with respect to excitability; the glucocorticoids lower threshold, and the mineralocorticoids elevate it (FIGURE 2). These findings provide a useful clinical tool for alteration of electrical threshold in implanted systems, and the use of prednisone to treat exit block is gaining acceptance. ${ }^{6}$ Less well established, and not as clinically important, is the use of a mineralocorticoid to raise threshold, or decrease myocardial responsiveness to an implanted pacemaker stimulus. This could conceivably be a very useful therapeutic adjunct in any condition in which there is a danger of the pacemaker stimulus producing a ventricular arrhythmia, as is possible following acute myocardial infarction, during competition immediately after pacemaker im-

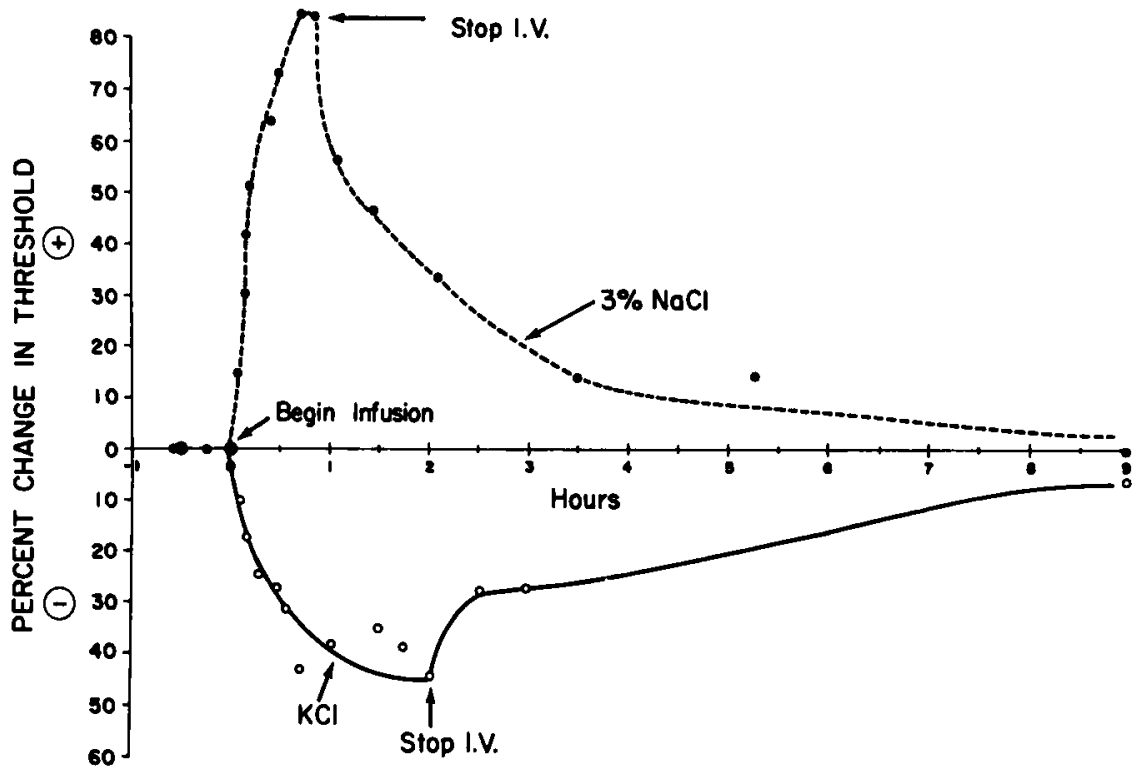

FIgure 1. Threshold changes after intravenous infusions of $\mathrm{NaCl}(3 \%)$ and $\mathrm{KCl}(50 \mathrm{mEq}$ in $500 \mathrm{ml}$ of Ringer's solution) in the same patient on different days. The $3 \% \mathrm{NaCl}$ was infused at a rate of 120 drops per minute, the $\mathrm{KCl}$ at 50 drops per minute. In each case, threshold remained constant for at least 30 minutes prior to the infusion. 


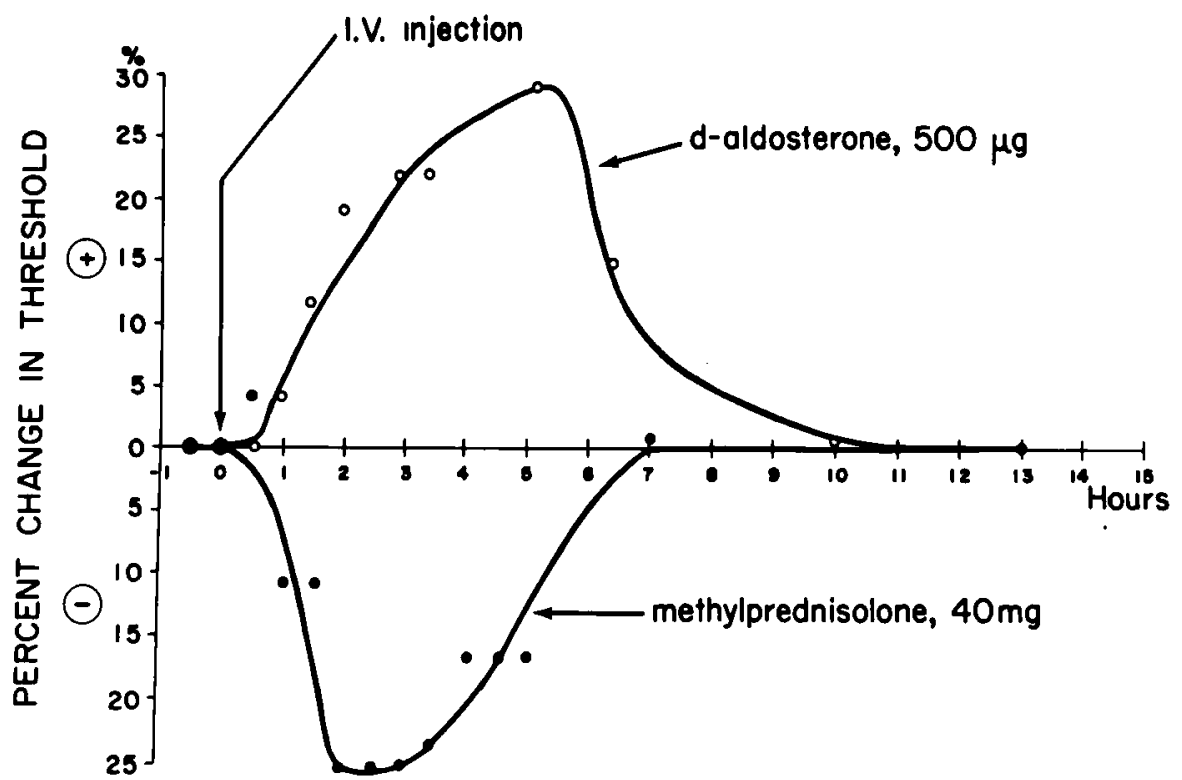

FIGURE 2. Changes in threshold after intravenous infusion of methylprednisolone and $d$-aldosterone in the same patient on different days.

plantation, and possibly even during the postimplant period with a demand pacemaker and no competition.

Because of the antagonism between the two types of steroids, therapy to alter threshold should employ either a pure glucocorticoid or pure mineralocorticoid. The drug effect appears to be dose related, and is not transient, since some patients have been treated for over a year with return of high threshold upon cessation of therapy. A mixed steroid such as hydrocortisone is likely to have an inconsistent effect on threshold.

Sympathomimetic agents in therapeutic doses increase excitability, and these agents are often the first used if a patient develops a high threshold. Ephedrine sulfate ( $25 \mathrm{mg}$ every six hours) will lower threshold by $10-20 \%$ in most cases. Oral isoproterenol $(10 \mathrm{mg})$ has a similar effect. In case of emergency, small doses of epinephrine or isoproterenol given intravenously result in lowered thresholds, but large intravenous doses of these drugs, given quickly, result in an initial lowering of the threshold, followed by a later elevation of threshold (FIGURE 3). This is a dose-related phenomenon, and smaller amounts of these sympathomimetics cause only a sustained threshold decrease. In our experience, all threshold alterations due to sympathomimetic amines can be abolished by the beta-blocking agent propranolol ( $1 \mathrm{mg}$, intravenously). Propranolol will elevate threshold if the patient has been physically active prior to testing, but we were unable to demonstrate an effect of propranolol on threshold in patients in a resting basal state. This is indirect evidence for the hypothesis that activity decreases threshold through sympathetic stimulation of the heart. In our experience, atropine has had no effect on electrical stimulation of the human ventricle.

The last group of drugs with which we are concerned, the antiarrhythmia agents, appears to have no effect on stimulation threshold. In studies on humans with complete heart block but otherwise normal hearts, we found no significant 


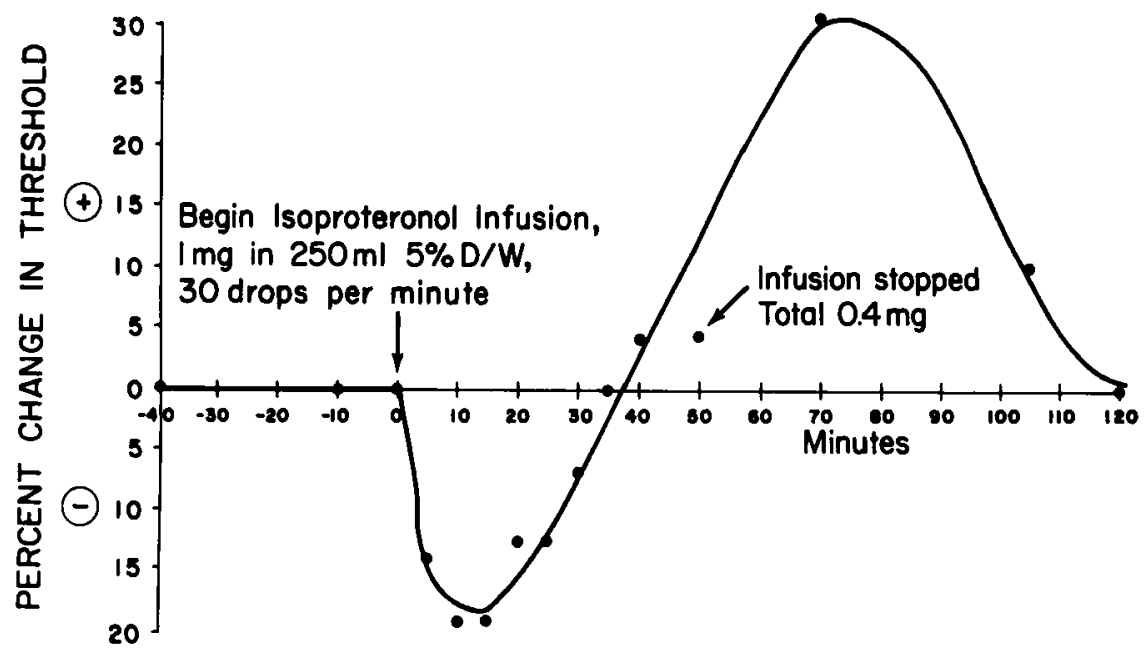

FIGURE 3. Effect of intravenous isoproteronol. Note initial decrease and then elevation of threshold.

alteration of threshold after therapeutic doses of quinidine, procainamide hydrochloride (900 mg in 55 minutes) and lidocaine $(1 \mathrm{mg} / \mathrm{kg}$, intravenously, in one dose). These findings correlate with reports that quinidine alters the cell membrane action potential but does not alter resting membrane potential in normal nonischemic cells. ${ }^{7}$ Since these agents did not affect the electrical threshold of the heart, under the conditions of our study it would appear that they could be used in controlling cardiac rhythm disorders in pacemaker patients whose arrhythmias are not related to pacemaker stimulation. In fact, we have observed abolition of premature ventricular beats with no change in threshold. The following case report is an example of the dynamic effect of various drugs on electrical threshold.

The patient was a 43-year-old female with acquired complete heart block, treated with an implanted pacemaker. Three years following the first implantation she developed headaches and nausea, and had a grand mal seizure. She was found to be hypertensive, and renal arteriograms showed segmental occlusion of the left renal artery. Renal endarterectomy was performed, for which anesthesia was induced with cyclopropane and continued for about one hour. At that time, the cyclopropane was stopped because of a potential

TABLE 2

Thresholds in a Patient before, during, and after Renal Endarterectomy

\begin{tabular}{lcc}
\hline \hline & $\begin{array}{c}\text { Threshold } \\
\text { (microjoules) }\end{array}$ & $\begin{array}{c}\text { Threshold } \\
\text { Change (\%) }\end{array}$ \\
\hline Baseline (no change over & & \\
48 hours before operation) & 8.5 & -44.7 \\
After 75 minutes of cyclopropane & 4.7 & -25.9 \\
After 30 minutes of halothane & 6.3 & -43.5 \\
Two hours after operation & 4.8 & -47.1 \\
Two days after operation & 4.5 & -4.5 \\
\hline
\end{tabular}


hazard produced by electronic equipment in the operating room, and halothane was substituted as the anesthetic. Threshold was monitored before anesthetic induction, during the operation, and until two days after the operation (TABLE 2). The threshold was very stable before the operation, but administration of cyclopropane caused a significant decrease in threshold. Following the switch from cyclopropane to halothane, the threshold rose significantly. This correlates with the known cardiac excitatory effects of cyclopropane and depressant effect of halothane. Following termination of the operation, with reinstitution of normal blood supply to the left kidney, the threshold slowly dropped to a level $47 \%$ below the preoperative baseline level. A possible explanation of the lowered postoperative threshold is that preoperatively the renal arterial obstruction resulted in activation of the renin system and increased the levels of aldosterone, which elevated cardiac electrical threshold. After removal of the obstruction, the hyperaldosterone state diminished to normal, with a resultant decrease in threshold. It is also possible that muscle-relaxing agents affect cardiac threshold, and there is a reported case in which electrical stimulation was apparently blocked by suxamethonium. ${ }^{8}$

In addition to exit block, there are other clinical conditions in which threshold alteration is useful. For the patient with early battery failure detected by changing pacemaker rate or by other methods, but without clinical failure of the system, there is always the question whether the power unit must be changed immediately or whether the safety factor is great enough to wait for regular scheduling of the surgical procedure. If the relative threshold can be measured, the safety factor is easily determined. If there is very little safety factor, short-term treatment with prednisone will lower the threshold relative to the already reduced stimulus, thus increasing the safety factor and allowing for regular surgical scheduling rather than an emergency procedure. Such treatment also allows time for a more careful assessment of the case and procurement of the exact type of pacemaker needed. In addition, if the threshold for a given system is known prior to battery failure, and has been demonstrated to be low, a low-energy unit can be used when replacement of the unit becomes necessary, providing there is a large enough safety factor. Such a low-energy unit should save battery drainage and increase pacemaker longevity.

In summary, controlled experiments have demonstrated that drugs can alter the reaction of human cardiac tissue to electrical stimuli. With future technical advances in external electronic manipulation of implanted pacing systems, drug therapy for threshold problems may become obsolete. New electrode designs should result in markedly lower energy thresholds, but the problem of relatively high thresholds and the finite ratio between fibrillation and excitation threshold will remain. Regardless of future clinical usefulness of drugs, however, the effects of these agents demonstrate the interaction between electromechanical and biological systems, and remind both the engineer and the physician that a design or therapeutic approach which disregards one or the other system will fall short of maximal potential.

\section{References}

1. Davies, J. G. \& G. E. Sowton. 1966. Electrical threshold of the human heart. Brit. Heart J. 28: 231 .

2. Preston, T. A., R. D. Judge, B. R. Lucchesi \& D. L. Bowers. 1966. Myocardial threshold in patients with artificial pacemakers. Amer. J. Cardiol. 18: 83-89.

3. Preston, T. A., R. D. Fletcher, B. R. Lucchesi \& R. D. JudGe. 1967. Changes in myocardial threshold. Amer. Heart J. 74: 235. 
4. Walker, W. J., J. T. Elkins \& L. W. Wood. 1964. Effect of potassium in restoring myocardial response to subthreshold cardiac pacemaker. New Eng. J. Med. 271: 597.

5. Principles of Internal Medicine. 5th edit. : 1326. McGraw-Hill Book Co. New York, N. Y. (1966).

6. Siddons, H. \& E. Sowton. 1967. Cardiac Pacemakers. : 169. Charles C Thomas, Publ. Springfield, Ill.

7. Gettes, L. S., B. Surawicz \& J. C. Shive. 1962. Effect of high K, low K, and quinidine on QRS duration and ventricular action potential. Amer. J. Physiol. 203: 1175.

8. Lawrence, G. H., R. M. Paine \& M. L. Hughes. 1965. Management of complications associated with the use of implantable electronic cardiac pacemakers for the relief of complete heart block. Amer. J. Surg. 110: 177-185. 